\title{
The Real-Time Mach-Zehnder Interferometer used in Space Experiment
}

\author{
L. Duan • Q. Kang $\cdot$ Z. W. Sun $\cdot$ L. Hu $\cdot$ H. L. Cui • \\ H. Lin • G. P. Li
}

Received: 10 January 2007 / Accepted: 27 November 2007 / Published online: 3 April 2008

(C) Springer Science + Business Media B.V. 2008

\begin{abstract}
The optical interference method is a promising technique for measuring temperature, density, and concentration in fluids. The non-intrusive and non-invasive nature of its optical techniques to the measured section are its most outstanding features. However, the adverse experiment environment, especially regarding shaking and vibrating, greatly restricts the application of the interferometer. In the present work, an optical diagnostic system consisting of a Mach-Zehnder interferometer (named after physicists Ludwig Mach) and an image processor has been developed that increases the measuring sensitivity compared to conventional experimental methods in fluid mechanics. An image processor has also been developed for obtaining quantitative results by using Fourier transformation. The present facility has been used in observing and measuring the mass transfer process of a water droplet in EAFP protein solution under microgravity condition provided by the satellite Shi Jian No. 8 .
\end{abstract}

Keywords Mach-Zehnder interferometer - Mass transfer. Diffusion

\section{Introduction}

Understanding much of the physics phenomena in fluid mechanics strongly depends on the development of measuring techniques in fluids. Mass transfer is one of the most important topics in microgravity fluid science. Diffusion is a concentration driven mass transfer process

L. Duan $(\bowtie) \cdot$ Q. Kang $\cdot$ Z. W. Sun $\cdot$ L. Hu .

H. L. Cui $\cdot$ H. Lin $\cdot$ G. P. Li

National Microgravity Laboratory/CAS, Institute of Mechanics,

Chinese Academy of Sciences,

Beijing 100080, China

e-mail: duanli@imech.ac.cn that takes place when the distribution of composition concentration in a solution is uneven. In normal gravity, the mass transfer due to diffusion is usually accompanied by mass transfer caused by convection and sedimentation. The diffusion coefficient of binary solutions is an important parameter in modeling studies of protein crystal growth. However, it is difficult to accurately measure it with ground-based experiments because of the influence of convection and sedimentation caused by gravity. This situation impels scientists to conduct experiments on the diffusion coefficient of binary solutions in a microgravity environment to eliminate the effect of sedimentation and convection. Among the related microgravity experiments, one is a space-flight experiment entitled, "The Dynamics of Miscible Interfaces: A Space Flight Experiment", which is financed by NASA. Another is "Diffusion and Soret Coefficients Measurement for Improvement of Oil Recovery", which is a part of "Microgravity Application Promotion" financed by the European Space Agency (ESA).

The diffusion mechanism and the influence of concentration gradient to diffusion coefficient have also been discussed (Eaton and Akgerman 1997; Fei and Bart 2001). Regarding the methodology of measurement, in recent years research efforts have mainly focused on measuring the diffusion process of liquid-liquid phase with the interferometric method (Elcloe 2000; Laurence and Jacques 2002). However, the recording method of holographic interferometry and the difficulty of confirming the second exposal time make this technique limited in its application to space experimentation. The real-time Mach-Zehnder interferometer will therefore take a key role in the study of diffusion mass transfer in microgravity that will apply to many other research fields.

The interference technique is based on the light-wave interference principle and has high a measuring sensitivity and veracity in comparison to other conventional optics 
methods. Its non-intrusive and non-invasive peculiarities to the measured section are its most outstanding features. Because the laser, the optoelectronic technology, and the computer technology achieve the phase subdivision with high resolving ability and veracity, measuring sensitivity and veracity of the interference technique have been significantly improved and its application range has also been extended, such as precision measurement, precision finishing, and real-time control surveying.

The interferometer can be classified into two major types according to the arrangement of its optical path: namely, the common-path interferometer and the two-path interferometer. The common-path interferometer is where the object light beam and reference light beam share a common path. The strong point of this kind of interferometer is that the interferometer can resist disturbance of the environment. $\mathrm{N}$. Rashidnia and R. Balasubramaniam designed a commonpath interferometer for use in space experiments (Rashidnia and Balasubramaniam 2004), and some experimental results in normal gravity have been obtained. The associated experimental facility is simple, non-maladjusted, and resists vibration, and is therefore suitable for space experimentation. One disadvantage, however, is its lower measurement accuracy and sensitivity compared to the twopath interferometer. In addition, its image processing is much more complicated in distinguishing information of object light beam and reference light beam because of the common path.

On the other hand, in the two-path interferometer, the object light beam and the reference light beam take two different paths. The influences of the vibrating environment to the two light beams are different. In interference measurement, in order to obtain stable interference fringes, some measuring conditions must be limited, and some protecting methods must be adopted, so this kind of interferometer is normally used in the ground-based laboratory, which has a stable environment.

The phase-shift Mach-Zehnder interferometer has been used as the "quasi-real-time" method to measure and visualize the change of the solution concentration during crystal growth and to study the heat and mass transfer by DUAN and KANG (Duan and Shu 2001; Kang and Duan 2001; Duan et al. 2002). This technique supports the observation of crystal growth together with fluid process, helping to understand the fluid-flow phenomena of the crystal-growth process. Using the Mach-Zehnder interferometer, the buoyancy convection (plume flow) during $\mathrm{NaClO}_{3}$ crystal growth (and the diffusion mass transfer during protein crystal growth) have been visualized and analyzed and the thickness of the diffusion layer were able to be measured accurately.

The Mach-Zehnder interferometer is a powerful tool for studying mass transfer, crystal growth, and other flowing processes. A German microgravity research center has designed a two-wavelength Mach-Zehnder interferometer that is applicable in the International Space Station (ISS). In ISS, the astronaut may operate the interferometer if the interferometer is maladjusted after the launch process.

However, obviously, there is no astronaut available on the satellite in our present space experiment, so an interferometer with the ability to resist vibration and shock during the launch phase of the rocket is desired in order to study mass transfer in space. As we know, there has not been any actual application of Mach-Zehnder interferometer in space experiment reported in the literature.

In this study, a specially designed Mach-Zehnder interferometer was used to monitor the mass transfer process of a water droplet in EAFP protein solution under both normal gravity and microgravity conditions provided by the satellite Shi Jian No. 8. A series of evolution charts of mass distribution during the diffusion process of the liquid droplet are presented. The optical diagnostic system consisting of a Mach-Zehnder interferometer enhances the measuring sensitivity in compared to the conventional experimental method in fluid mechanics, and the image processor has been developed for obtaining quantitative results. This optical interferometer has proven to be a good measurement method to study mass transfer process.

\section{The Optics Diagnostic Method}

\section{Basic Optics Theory}

A diagram of the optical diagnostic system is shown in Figs. 1 and 2. A laser beam passes through lens L1 and L2 to form an expanded parallel light beam, and then the beam is split by splitter Bs1 into two parallel light beams; one is used as the object beam and the other is used as a reference beam. The object light beam is reflected by mirror M2 firstly, and passes through liquid cell, and then passes through beam splitter Bs2. The reference light beam is reflected by mirror $\mathrm{M} 1$, and then reflected by beam splitter Bs2. At this time, the object beam and the reference beam meet and form an interferometric fringe pattern. The fringes carry the information related to a concentration gradient of

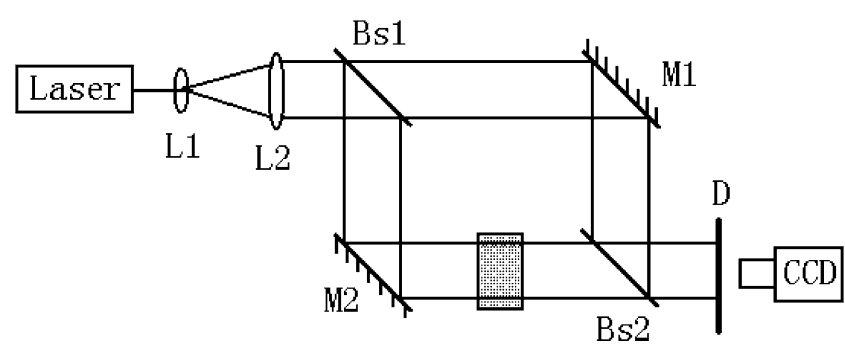

Fig. 1 Mach-Zehnder interferometer 


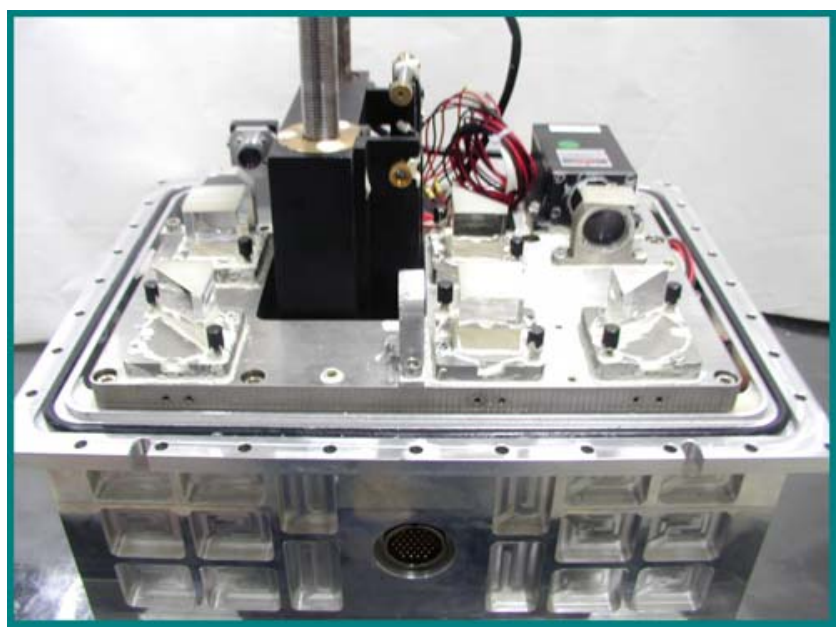

Fig. 2 The space experimental facility

solution flow field in liquid cell, which are then captured by a CCD camera.

Two coherent light beams meet and then interferometric fringes appear as a result of the interference. These fringes carry the information associated with the characteristics of flow field in which the light passes through. Supposing that $A_{0}(x, y)$ and $A_{1}(x, y)$ are complex amplitudes of two light beams, which have the same vibration direction, equal frequency, and transmit in the $x-y$ plane; the two complex amplitudes are expressed as follows:

$A_{i}(x, y)=a_{i}(x, y) \exp \left[j \phi_{i}(x, y)\right], \quad(i=0,1)$.

where $\phi_{i}(x, y)$ is the optical phase determined by the wavelength of light $\lambda$ and optical distance $L_{i}(x, y)$ as

$\phi_{i}(x, y)=\frac{2 \pi}{\lambda} L_{i}(x, y), \quad(i=0,1)$.

Superposition light intensities of the two light beams are

$$
\begin{aligned}
I(x, y) & =\left|A_{0}(x, y)+A_{1}(x, y)\right|^{2} \\
& =I_{0}(x, y)+I_{c}(x, y) \cos \Delta \phi(x, y),
\end{aligned}
$$

here

$I_{0}(x, y)=a_{0}^{2}(x, y)+a_{1}^{2}(x, y)$,

$I_{c}(x, y)=2 a_{0}(x, y) a_{1}(x, y)$,

$\Delta \phi(x, y)=\phi_{1}(x, y)-\phi_{0}(x, y)$.

Interference fringes will occur when the two light beams have been superposed. The fringes hold information on amplitude and phase, and the phase which expresses the difference of light distance is calculated by analyzing the interference fringes.
The Experiment Facility

The facility used to study mass transfer on the satellite Shi Jian No. 8 is composed of four substantive parts as follows:

A. A Mach-Zehnder interferometer, including a laser, two lenses, two beam-split prisms, three triangle prisms, and a screen.

B. An image capturing and processor system, including a CCD camera and an image-capture card.

C. A liquid cell system that holds diffusion liquid samples.

D. An operation controlling system.

The experimental facility was supported by a $28-\mathrm{V}$ power supply of the satellite system. The CCD camera, the laser, and the motor were powered, respectively, by 9,5 , and $24-\mathrm{V}$ second power supplies. When the motor was working, the average power was $24 \mathrm{~W}$. When the motor was running, the power was $29 \mathrm{~W}$. The material of the facility harness was anodized aluminum. The weight of the entire facility was $27.2 \mathrm{~kg}$, with a volume of $350 \times 280 \times 360 \mathrm{~mm}^{3}$. The facility was operated by the telecontrol dictates combining with the program-control dictates. The CCD camera captured the images at a rate of 5 frames per second.

The Mach-Zehnder interferometer is a two-path optics measurement system, which has high accuracy. It has been used only in a laboratory that is basically vibration-free and shock-free. The environmental factors affecting the interferometer's accuracy include air disturbance, mechanical instability, and thermal instability. The interferometer is usually set up on an anti-vibration optical table to eliminate environmental disturbances and for operation in a constant-temperature condition. The Mach-Zehnder interferometer is both very sensitive and accurate, but it is also easily maladjusted by vibration, shock, and temperature changes. To prevent the Mach-Zehnder interferometer from being maladjusted, some special anti-vibration measures were taken. For instance, a stainless-steel board with a thickness of $14 \mathrm{~mm}$ was used as optics soleplate, upon which all three-dimensional adjustors were firmly locked after the optical modulating procedure. In this operation, mirrors are replaced by triangle prisms and beam splitters are replaced by splitter prisms in order to increase the contact area between the optics glasses and the optics soleplate. In addition, the optics glasses and the optics soleplate have been firmly glued. The complete specially designed interferometer system (including optics system and image-capturing system) withstood the serious tests imposed during the rocket-launching phase and in the space environment and demonstrated an excellent performance.

\section{Calculation of Interference Fringes}

In order to calculate the phase difference from interference fringes, grating analysis was used. The fringe before mass 


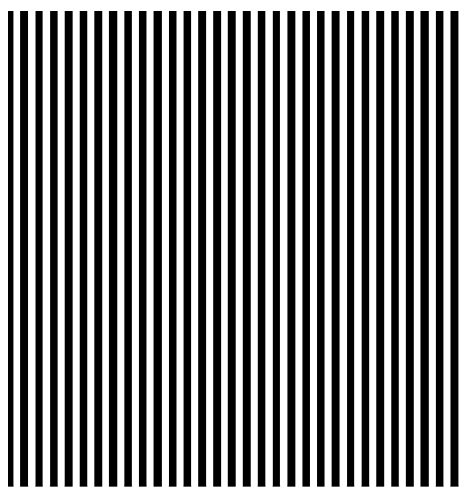

a

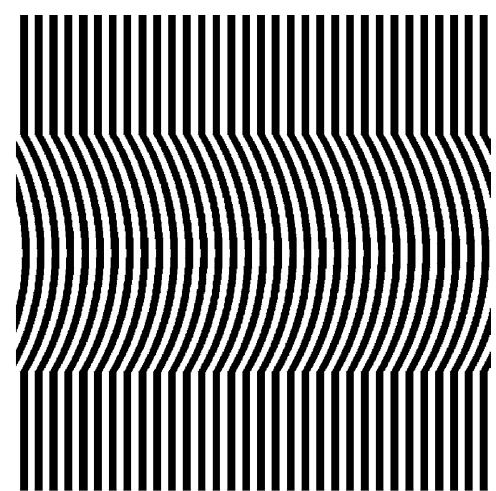

b

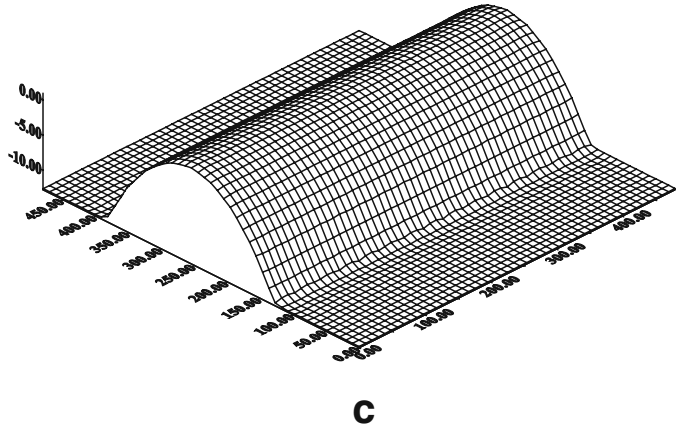

Fig. 3 The calculation of the simulating fringes

transfer was used as the original grating, and in this case, the change of refractive index $\Delta n(x, y)=0$. The image can be expressed as Fourier series $g_{0}(x, y)$, and the fringe for obtaining the change of refractive index was as the metamorphic grating, here, the change of refractive index $\Delta n(x, y) \neq 0$, the image can be expressed as Fourier series $g(x, y)$.

$$
\begin{aligned}
\Delta n(x, y) & =0, g_{0}(x, y) \\
& =r_{0}(x, y) \sum_{n=-\infty}^{\infty} A_{n} \exp \left\{i\left[2 \pi n f_{0} x+n \phi_{0}(x, y)\right]\right\} \\
\Delta n(x, y) & \neq 0, g(x, y) \\
& =r(x, y) \sum_{n=-\infty}^{\infty} A_{n} \exp \left\{i\left[2 \pi n f_{0} x+n \phi(x, y)\right]\right\}
\end{aligned}
$$

Here, $r(x, y)$ stands for the asymmetric disturbance of reflectivity, $f_{0}$ stands for basal frequency, $\phi(x, y)$ stands for phase. We chose $n=1$, and calculated the cross-Fourier transformation. The phase distributions of the metamorphic grating and the original grating were obtained as follows:

$\widehat{g}(x, y)=A_{1} r(x, y) \exp \left\{i\left[2 \pi f_{0} x+\phi(x, y)\right]\right\}$

$\widehat{g}_{0}(x, y)=A_{1} r_{0}(x, y) \exp \left\{i\left[2 \pi f_{0} x+\phi_{0}(x, y)\right]\right\}$

$\widehat{g}(x, y) \widehat{g}_{0}^{*}(x, y)=\left|A_{1}\right|^{2} r_{0}(x, y) r(x, y) \exp \{i[\Delta \phi(x, y)]\}$

$\Delta \phi(x, y)=\phi(x, y)-\phi_{0}(x, y)$

The change of refractive index $\Delta n(x, y)$ was calculated by the relationship between phase difference $\Delta \phi$ and $\Delta n(x, y)$.

$\frac{\Delta n(x, y) \cdot d}{\lambda} \cdot 2 \pi=\Delta \phi(x, y)$

$\Delta n(x, y)=\frac{\Delta \phi(x, y) \cdot \lambda}{2 \pi \cdot d}$

$n(x, y)=n_{0}+\Delta n(x, y)$

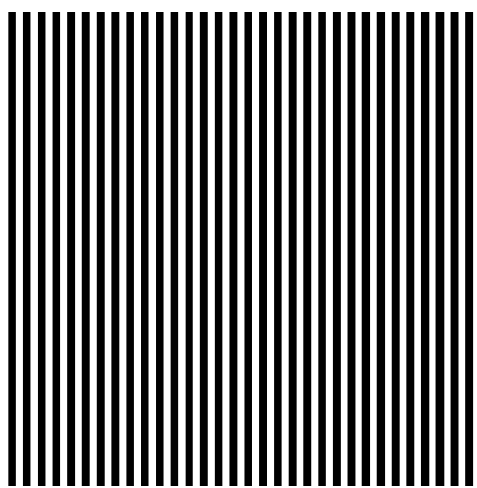

a

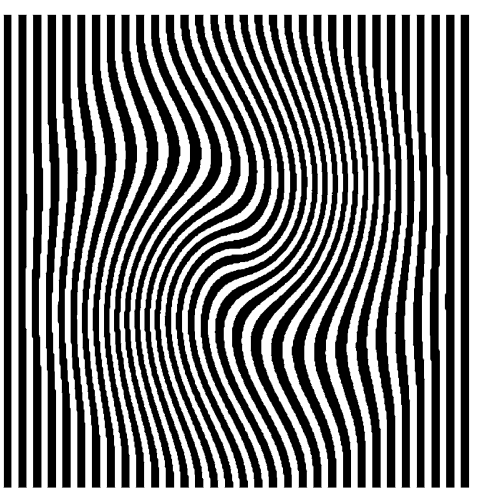

b

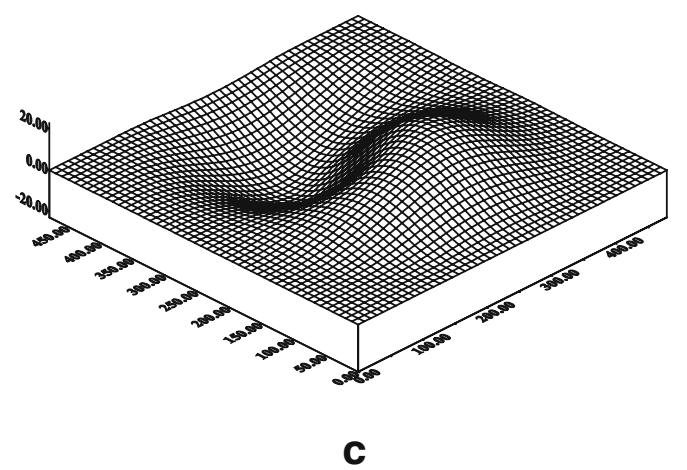

Fig. 4 The calculation of the simulating fringes 

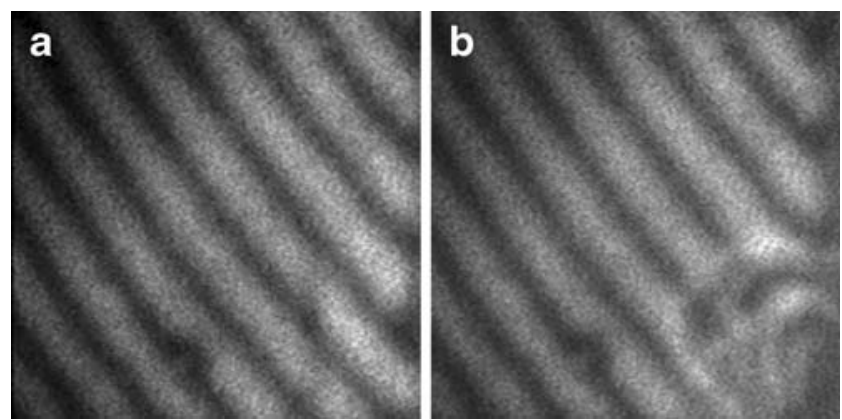

Fig. 5 Before (a) and just after (b) the water droplet was injected

The relationship between the refractive index and the concentration of EAFP protein solution was measured by WAY-15 ABBE REFRACTOMETER in order to convert the refractive index field into a concentration field; the relative formula can be written as follows.

$C=1000.07 \cdot n-1325.5$

where, $n$ and $C$ are the refractive index and concentration of EAFP protein solution, respectively.

According to this image-processing method, two simulation fringes have been illustrated, which proved that the image-processing method is reasonable. Figure $3 \mathrm{a}$ is the original fringe pattern and Fig. $3 b$ is the metamorphic fringe pattern. The quantitative result of cylindrical surface has been calculated as shown in Fig. 3c. A similar calculation is shown in Fig. 4.

\section{The Mechanics Environment Experiments}

The present experiment facility has been tested by the mechanics environment experiments. The test items included sine shaking, stochastic vibration, impact test, and acceleration test. The 50-KN electric vibrated table of ES50000 model, $49-\mathrm{KN}$ electric vibrated table of M1216VH model, and 43 model centrifugal machine were used in the test. The temperature of the environment was $25^{\circ} \mathrm{C}$. The test parameters and the results are expressed in the tables below.

The facility has passed the leak test under the condition of vacuum. A ZQJ-230D helium mass spectrometer was used to examine the leak by spewing helium. The space experiment facility was evacuated to vacuum level under the value of $5 \mathrm{~Pa}$. The link components were spewed by helium and then the leaking rate was measured. The result is that the leaking rate is $1.2 \times 10^{-9} \mathrm{~Pa} \cdot \mathrm{m}^{3} / \mathrm{s}$. The facility has also been tested in the environment of heat circulation. The high temperature of the working environment is $40^{\circ} \mathrm{C}$, and the low temperature is $-5^{\circ} \mathrm{C}$; the facility worked well.

\section{The Space Experiment Results and the Quantitative Calculation}

The diffusion process of a water droplet immersed in EAFP protein solution was observed in the space experiment on the satellite Shi Jian No. 8 launched in 2006. Since the

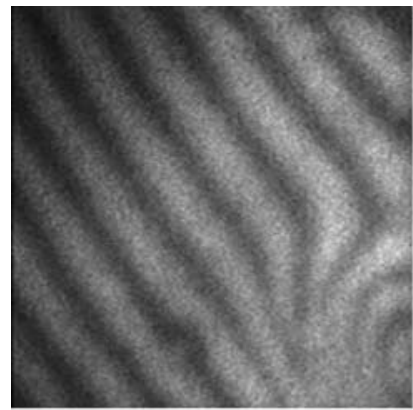

$\mathbf{a}_{\mathrm{t}}=304.5 \mathrm{~s}$

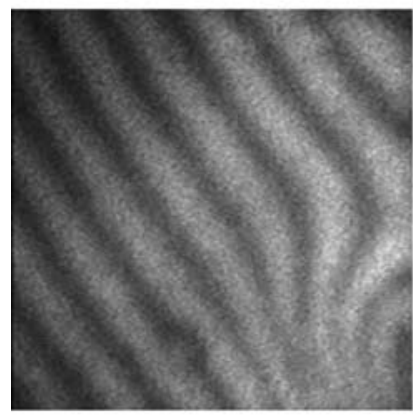

C $\mathrm{t}=548.7 \mathrm{~s}$

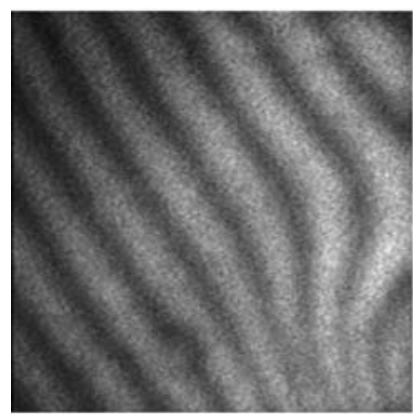

e $\mathrm{t}=906.0 \mathrm{~s}$

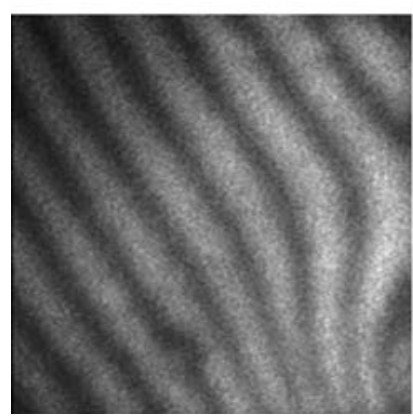

$\mathbf{g} \mathrm{t}=1271.0 \mathrm{~s}$

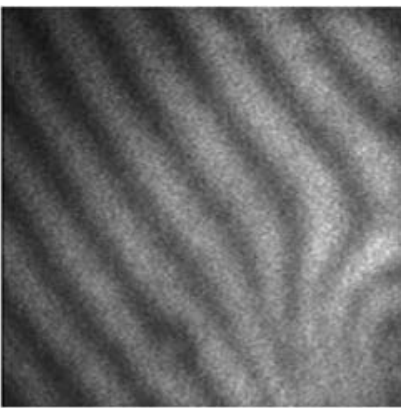

b $t=430.4 s$

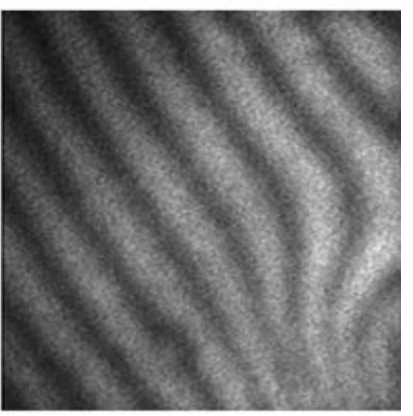

d $\mathrm{t}=724.8 \mathrm{~s}$

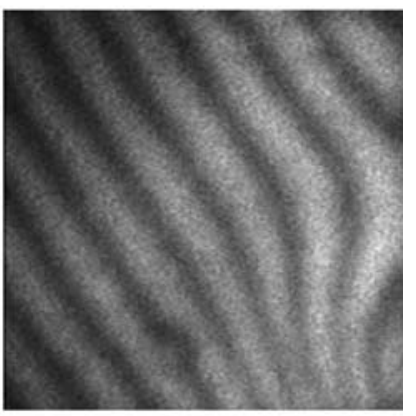

$f t=1087.3 \mathrm{~s}$

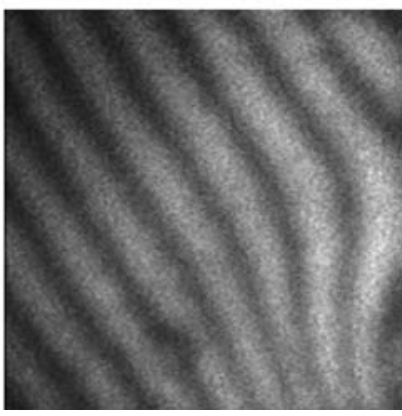

h $\mathrm{t}=1449.7 \mathrm{~s}$
Fig. 6 The interference fringes in space experiment 
Fig. 7 The concentration distribution calculated from interference fringes
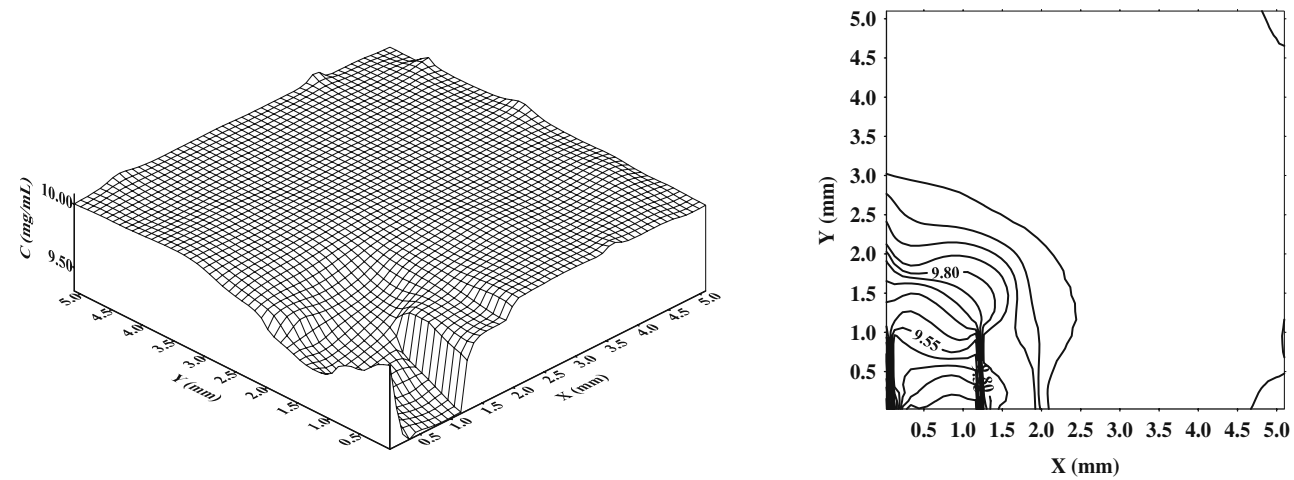

a $\mathrm{t}=304.5 \mathrm{~s}$

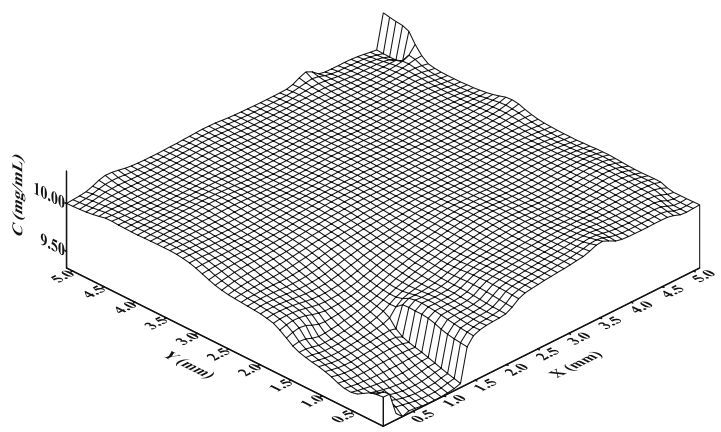

b $\mathrm{t}=724.8 \mathrm{~s}$
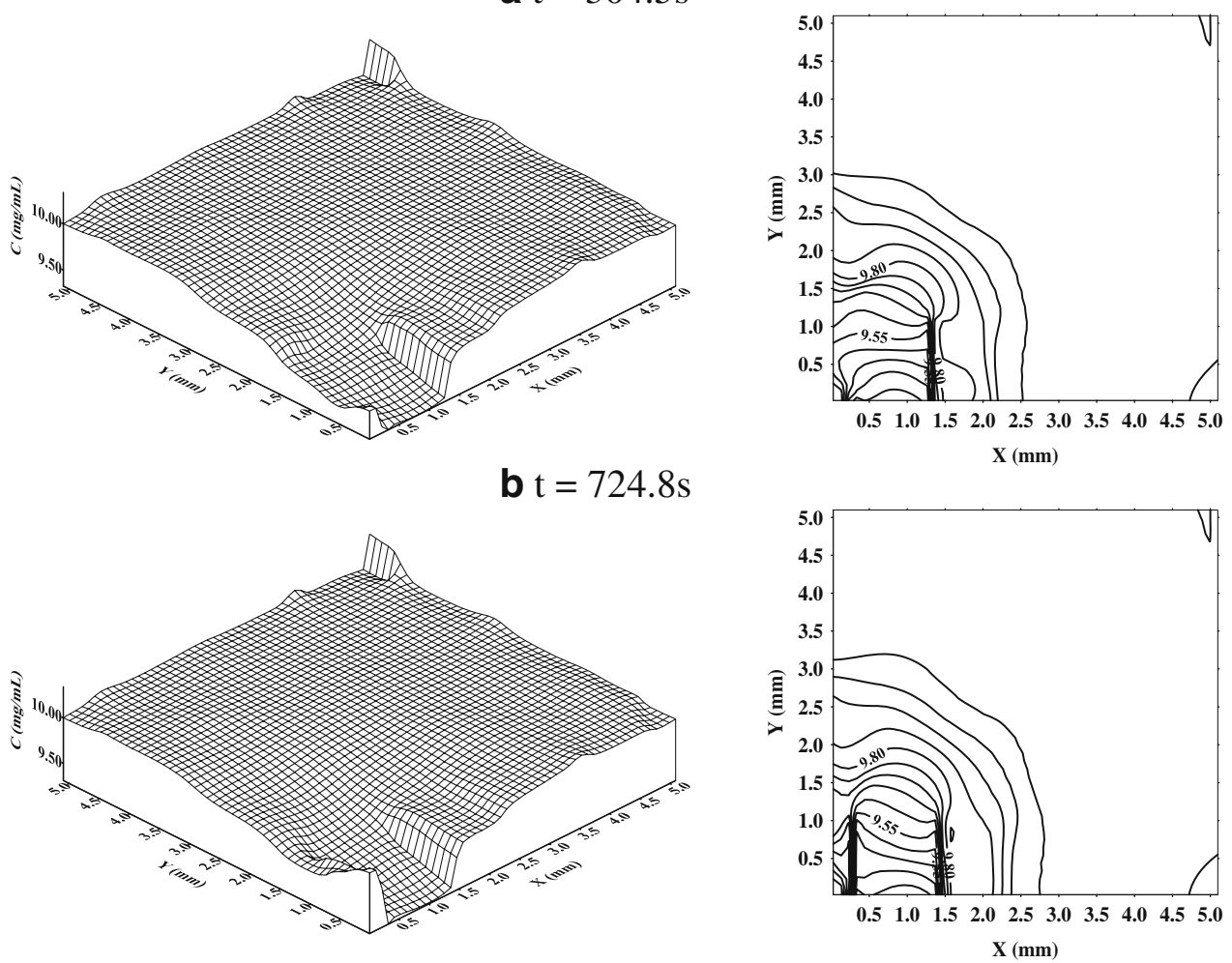

$\mathbf{C} \mathrm{t}=1087.3 \mathrm{~s}$
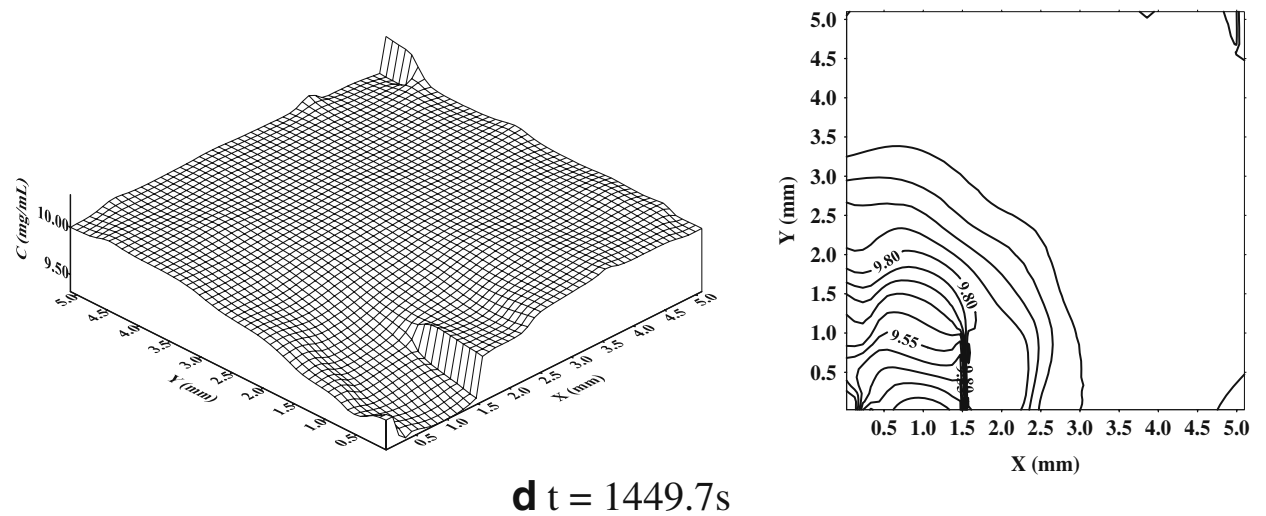

contribution from convection and sedimentation were excluded in microgravity, the mass transfer was caused only by a pure diffusion process. An EAFP protein solution with a concentration of $10 \mathrm{mg} / \mathrm{mL}$ was contained in a cell with the dimensions of $6 \times 6 \times 1.5 \mathrm{~mm}$. In order to calculate the concentration gradient of the diffusion process, a water droplet with a much smaller size $(0.15 \mathrm{~mm})$ was injected into the cell at the bottom. 
Fig. 8 The concentration distribution at three directions

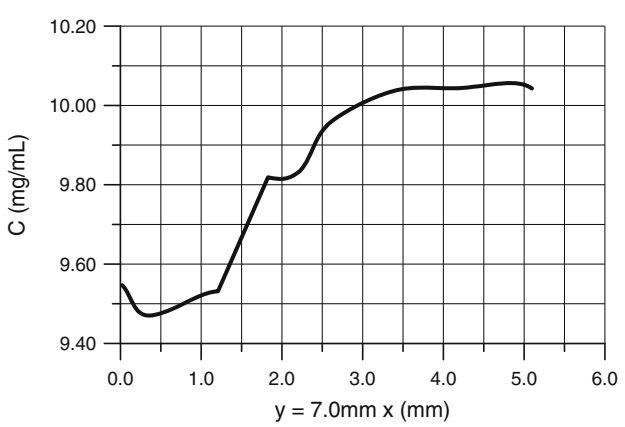

a The concentration distribution along $\mathrm{x}$ direction

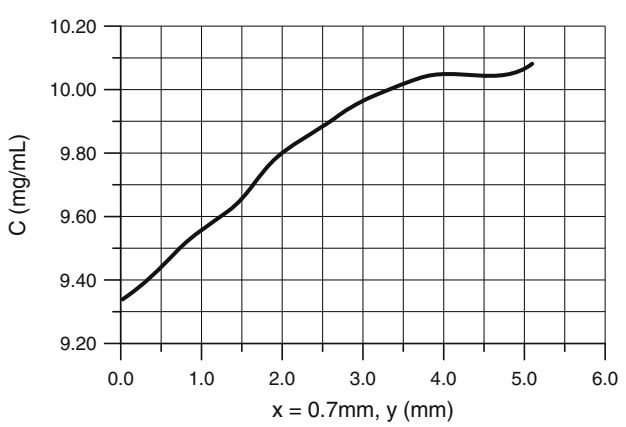

b The concentration distribution along y direction

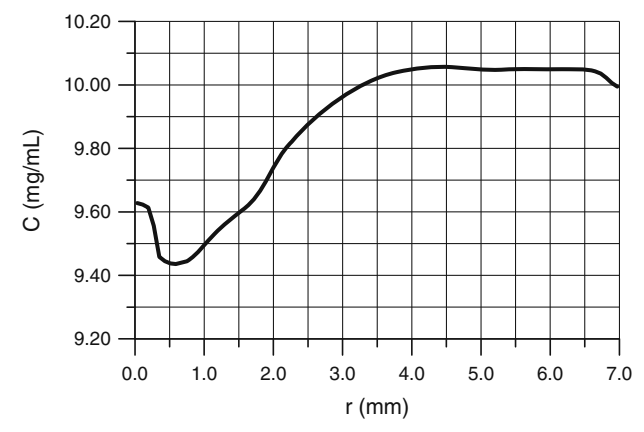

C The concentration distribution along $\mathrm{R}$ direction

Because of the much smaller water droplet and the rapid diffusion process, there is no obvious borderline between the water droplet and the protein solution, but the concentration gradient can be measured and calculated and therefore the diffusion mass transfer can be estimated according to the changed interference fringes. Figure 5 shows the images where (a) is the before the water droplet was injected into the protein solution and (b) is just after the water droplet was injected.

For measuring the concentration gradient by MachZehnder interferometer, the measured quantity should be

Table 1 Test parameters and results of sine shaking test

\begin{tabular}{ll}
\hline Test item & The test of sine scan \\
\hline Scan time & $55 \mathrm{~s} /$ direction \\
Loading direction & $X, Y, Z$ directions \\
Frequency $(\mathrm{Hz})$ & The maximum is $100 \mathrm{~Hz}$ \\
Vibration magnitude & The maximum is $11.5 \mathrm{~g}$ \\
\hline
\end{tabular}

Table 2 The test parameters and results of test stochastic vibration

\begin{tabular}{ll}
\hline Test item & The test of stochastic vibration \\
\hline Loading time & $60 \mathrm{~s} /$ direction \\
Loading directions & $X, Y, Z$ directions \\
Frequency $(\mathrm{Hz})$ & The test range is 10 to $2,000 \mathrm{~Hz}$ \\
Vibration magnitude & The root-mean-square acceleration \\
& magnitude is $10 \mathrm{~g}$ \\
\hline
\end{tabular}

nearly constant along the light direction, so a sample cell with the dimensions of $6 \times 6 \times 1.5 \mathrm{~mm}$ was adopted. Here, the thickness of the cell is $1.5 \mathrm{~mm}$, which is the distance that the light travels. The interference fringes reveal the integral results of the light across the $1.5-\mathrm{mm}$ distance, and the calculation ignores the difference of the concentration along the path of the light, which is a usual method for the experiment measured by the interferometer.

In the microgravity environment, the diffusion process should be spherically symmetric when the concentration

Table 3 The test parameters and results of the impact test

\begin{tabular}{ll}
\hline Test item & The impact test \\
\hline Impact times & 2times/direction \\
Loading directions & $X, Y, Z$ directions \\
Frequency (Hz) & The test range is 100 to $3,000 \mathrm{~Hz}$ \\
The magnitude of impact & $+6 \mathrm{db} /$ oct $600 \mathrm{~g}$ \\
spectrum & \\
\hline
\end{tabular}

Table 4 The test parameters and results of the acceleration test

\begin{tabular}{ll}
\hline Test item & The acceleration test \\
\hline Loading time & $120 \mathrm{~s}$ \\
The acceleration change rate & $\leq 0.5 \mathrm{~g} / \mathrm{s}$ \\
The acceleration magnitude & The maximum is $10.35 \mathrm{~g}$
\end{tabular}


gradients along all directions are the same. Figure 6 shows a series of photos of interference fringes taken at different times, which clearly show a steady diffusion process. We also can see that the shape of the droplet was well was maintained for a long period. The whole field concentration distribution at different times was calculated from interference fringes according to basic optics theory. The concentration distributions are shown in Fig. 7. The concentration changes obviously, and the contour map is concentric circles at the water droplet region. The concentration changes smoothly and gradually from the water drop. It demonstrates that mass transfer in microgravity is a pure diffusion process.

Based on the analyses of the experimental data, concentration distributions along the $x$-direction, $y$-direction, and $\mathrm{R}$ $\left(R=\sqrt{X^{2}+Y^{2}}\right)$ direction at the time of $t=1328.9 \mathrm{~s}$ are given in Fig. 8. These three figures show that the mass transfer in all directions extended to a distance of $4 \mathrm{~mm}$ until the water drop immersed, and the diffusion speeds seemed to be almost even in all directions.

Because of the scarce space experiment opportunity, only the results of one experiment have been obtained. However, the results we obtained can stand for the typical diffusion behavior as the diffusion process under microgravity condition is theoretically induced by the concentration gradient (see Tables 1, 2, 3, and 4).

\section{Conclusions}

We demonstrate that a well-designed Mach-Zehnder interferometer is able to be used in space experiments facing challenges from the serious tests of violent shock and vibration during the rocket-launching phase. The whole diffusion process of a water droplet in EAFP protein solution in microgravity was successfully observed by means of this interferometer in the satellite Shi Jian No. 8. The relevant features of the diffusion of the water droplet are analyzed and discussed according to changes of the interference fringes.

Acknowledgements This work was supported by the National Nature Science Foundation of China $(10432060,10672171)$ and the Knowledge Innovation Program of the Chinese Academy of Sciences (KACX2-SW-02, KSCX2-SW-322).

\section{References}

Duan, L., Kang, Q., Hu, W.R., Li, G.P., Wang, D.C.: Mass transfer process and growth rate of protein crystal growth. Biophys. Chemist. 97, 189-201 (2002) SCI 1.759

Duan, L., Shu, J.Z.: The convection during $\mathrm{NaClO}_{3}$ crystal growth observed by the phase shift interferometer. J. Cryst. Growth. 223, 181-188 (2001) SCI 1.5

Eaton, A.P., Akgerman, A.: Infinite-dilution diffusion coefficients in supercritical fluids. Ind. Eng. Chem. Res. 36, 923-931 (1997)

Elcloe, M.: Interferometric measurement of diffusion coefficients through a scanning laser beam. Opt. Eng. 39, (3), 744-750 (2000)

Fei, W.Y., Bart, H.J.: Predicting diffusivities in liquids by the group contribution method. Chem. Eng. 40, (6), 531-535 (2001)

Kang, Q., Duan, L.: Mass transfer process during the $\mathrm{NaClO}_{3}$ crystal growth process. Int. J. Heat Mass Transfer. 44, 3213-3222 (2001) SCI 0.7

Laurence, R., Jacques, B.: Influence of conformational changes on diffusion properties of bovine serum albumin: a holographic interferometry study. Colloid Surf. B: Biointerfaces. 25, (10), 99108 (2002)

Rashidnia, N., Balasubramaniam, R.: Measurement of the mass diffusivity of miscible liquids as a function of concentration using a common path shearing interferometer. Exp. Fluids. 36, 619-626 (2004) 